University of Nebraska - Lincoln

DigitalCommons@University of Nebraska - Lincoln

4-2013

\title{
Activation of interspecies-hybrid Rubisco enzymes to assess different models for the Rubisco-Rubisco activase interaction
}

Rebekka M. Wachter

Arizona State University

Michael E. Salvucci

USDA-ARS, mike.salvucci@ars.usda.gov

A. Elizabete Carmo-Silva

USDA-ARS

Csengele Barta

Missouri Western State University

Todor Genkov

University of Nebraska-Lincoln

See next page for additional authors

Follow this and additional works at: https://digitalcommons.unl.edu/usdaarsfacpub

Wachter, Rebekka M.; Salvucci, Michael E.; Carmo-Silva, A. Elizabete; Barta, Csengele; Genkov, Todor; and Spreitzer, Robert, "Activation of interspecies-hybrid Rubisco enzymes to assess different models for the Rubisco-Rubisco activase interaction" (2013). Publications from USDA-ARS / UNL Faculty. 1325. https://digitalcommons.unl.edu/usdaarsfacpub/1325

This Article is brought to you for free and open access by the U.S. Department of Agriculture: Agricultural Research Service, Lincoln, Nebraska at DigitalCommons@University of Nebraska - Lincoln. It has been accepted for inclusion in Publications from USDA-ARS / UNL Faculty by an authorized administrator of DigitalCommons@University of Nebraska - Lincoln. 


\section{Authors}

Rebekka M. Wachter, Michael E. Salvucci, A. Elizabete Carmo-Silva, Csengele Barta, Todor Genkov, and Robert Spreitzer 


\title{
Activation of interspecies-hybrid Rubisco enzymes to assess different models for the Rubisco-Rubisco activase interaction
}

\author{
Rebekka M. Wachter • Michael E. Salvucci • \\ A. Elizabete Carmo-Silva $\cdot$ Csengele Barta $\cdot$ \\ Todor Genkov $\cdot$ Robert J. Spreitzer
}

This article is a U.S. government work, and is not subject to copyright in the United States.

Received: 25 February 2013/Accepted: 8 April 2013

(C) Springer Science+Business Media Dordrecht (outside the USA) 2013

\begin{abstract}
Ribulose-1,5-bisphosphate carboxylase/oxygenase (Rubisco) is prone to inactivation from non-productive binding of sugar-phosphates. Reactivation of Rubisco requires conformational remodeling by a specific chaperone, Rubisco activase. Rubisco activase from tobacco and other plants in the family Solanaceae is an inefficient activator of Rubisco from non-Solanaceae plants and from the green alga Chlamydomonas reinhardtii. To determine if the Rubisco small subunit plays a role in the interaction with Rubisco activase, a hybrid Rubisco (SSNT) composed of tobacco small subunits and Chlamydomonas large subunits was constructed. The SSNT hybrid, like other hybrid Rubiscos containing plant small subunits, supported photoautotrophic growth in Chlamydomonas, but growth in air was much slower than for cells containing wild-type Rubisco. The kinetic properties of the SSNT hybrid Rubisco were similar to the wild-type enzyme, indicating that the poor growth in air was probably caused by disruption of pyrenoid formation and the consequent impairment of the $\mathrm{CO}_{2}$ concentrating mechanism. Recombinant Rubisco activase from Arabidopsis activated the SSNT hybrid Rubisco and hybrid Rubiscos containing spinach and Arabidopsis small subunits
\end{abstract}

R. M. Wachter

Department of Chemistry and Biochemistry, Arizona State

University, Tempe, AZ 85287, USA

M. E. Salvucci ( $₫)$ · A. E. Carmo-Silva · C. Barta

US Arid-Land Agricultural Research Center, Agricultural

Research Service, U.S. Department of Agriculture (USDA-ARS),

21881 N. Cardon Lane, Maricopa, AZ 85138, USA

e-mail: mike.salvucci@ars.usda.gov

Present Address:

A. E. Carmo-Silva

Plant Biology and Crop Science Department, Rothamsted

Research, West Common, Harpenden, Herts AL5 2JQ, UK at rates similar to the rates with wild-type Rubisco. However, none of the hybrid Rubiscos was activated by tobacco Rubisco activase. That replacement of Chlamydomonas small subunits with plant small subunits does not affect the species-specific interaction between Rubisco and Rubisco activase suggests that the association is not dominated by the small subunits that surround the Rubisco central solvent channel. Therefore, the geometry of a side-on binding mode is more consistent with the data than a top-on or ring-stacking binding mode.

Keywords $\mathrm{CO}_{2}$ fixation $\cdot$ Molecular chaperone $\cdot$ Proteinprotein interaction - Conformational remodeling .

Chlamydomonas $\cdot$ Chloroplast
Abbreviations
AAA + ATPase associated with diverse cellular activities
ER Rubisco in the uncarbamylated form and containing bound RuBP
ECM Rubisco carbamylated and containing bound $\mathrm{Mg}^{2+}$ $K_{\mathrm{c}} \quad K_{\mathrm{m}}$ for $\mathrm{CO}_{2}$

Present Address:

C. Barta

Department of Biology, Missouri Western State University, 4525

Downs Drive, Agenstein Hall 237G, Saint Joseph, MO 64507,

USA

T. Genkov $\cdot$ R. J. Spreitzer

Department of Biochemistry, University of Nebraska, Lincoln, NE 68588, USA

Present Address:

T. Genkov

Center for Biocatalysis and Bioprocessing, University of Iowa,

Coralville, IA 52241, USA 


$\begin{array}{ll}K_{\mathrm{o}} & K_{\mathrm{m}} \text { for } \mathrm{O}_{2} \\ \text { RuBP } & \begin{array}{l}\text { Ribulose 1,5-bisphosphate } \\ \text { Rubisco }\end{array} \\ \text { Ribulose-1,5-bisphosphate carboxylase/oxygenase } \\ \text { SSAT } & \begin{array}{l}\text { Hybrid Rubisco composed of Chlamydomonas } \\ \text { large subunits and Arabidopsis small subunits }\end{array} \\ \text { SSNT } & \begin{array}{l}\text { Hybrid Rubisco composed of Chlamydomonas } \\ \text { large subunits and tobacco small subunits }\end{array} \\ \text { SSSO } & \begin{array}{l}\text { Hybrid Rubisco composed of Chlamydomonas } \\ \text { large subunits and spinach small subunits }\end{array} \\ V_{\mathrm{c}} & \begin{array}{l}V_{\max } \text { of carboxylation } \\ V_{\mathrm{o}}\end{array} \\ \Omega & V_{\max } \text { of oxygenation } \\ \mathrm{CO}_{2} / \mathrm{O}_{2} \text { specificity factor }\end{array}$

\section{Introduction}

Ribulose-1,5-bisphosphate carboxylase/oxygenase (Rubi$\mathrm{sco}$ ) is the enzyme in photosynthetic organisms that converts atmospheric $\mathrm{CO}_{2}$ into organic matter (Spreitzer and Salvucci 2002; Whitney et al. 2011). The enzyme evolved early in earth's biotic history when the atmosphere contained high concentrations of $\mathrm{CO}_{2}$ and very little $\mathrm{O}_{2}$ (Blankenship 2002). As the atmosphere changed over the eons to one that is very enriched in $\mathrm{O}_{2}$ and with 1,000times less $\mathrm{CO}_{2}$ than the ancient atmosphere, a number of structural modifications evolved in Rubisco that are necessary for the enzyme to function in present day land plants (Tcherkez et al. 2006; Andersson and Backlund 2008). These modifications increased the binding affinity for ribulose 1,5-bisphosphate (RuBP) (Jordan et al. 1983), and promoted the production of inhibitory sugar-phosphates derived from reaction intermediates (Pearce and Andrews 2003; Pearce 2006). As a consequence, Rubisco requires conformational remodeling by a specific chaperone known as Rubisco activase. Rubisco activase "activates" Rubisco by relieving the dead-end inhibition caused by non-productive binding of the substrate or catalytic misfire products (Andrews 1996; Spreitzer and Salvucci 2002; Portis 2003).

Rubisco activase and $\mathrm{CbbX}$, its counterpart in red algae and proteobacteria (Mueller-Cajar et al. 2011), are AAA+ ATPases that appear to function as hexameric rings (Stotz et al. 2011), although they often occur as much larger oligomers (Barta et al. 2010; Chakraborty et al. 2012; Henderson et al. 2013). The plant enzyme exhibits a very peculiar type of species-specificity; Rubisco activase from members of the Solanaceae family (i.e., tobacco, tomato, petunia) are inefficient activators of Rubisco from nonSolanaceae plants, including the green alga Chlamydomonas reinhardtii, and vice versa (Wang et al. 1992). Previous studies with Chlamydomonas, spinach, and tobacco have identified two Rubisco residues (Larson et al.
1997; Ott et al. 2000) and a corresponding residue pair of Rubisco activase ( $\mathrm{Li}$ et al. 2005) that determine this specificity. The recognition-residues of Rubisco are at the surface of the large subunit and are located on the holoenzyme at an equatorial position remote from the active-site (Fig. 1) (Larson et al. 1997; Portis 2003). The corresponding Rubisco activase residues reside within the sensor- 2 region of the $\alpha$-domain of the AAA + module (Portis et al. 2008). Based on the X-ray crystal structure of Rubisco activase from creosote (Larrea tridentata), a nonSolanaceae species, the two specificity-determining residues occur at or near the end of a long $\alpha$-helix that extends out from the $\alpha$-domain (Henderson et al. 2011). In tobacco, this long helix is replaced by two $\alpha$-helices connected by a short loop (Stotz et al. 2011); the two tobacco residues that affect specificity occur on the smaller, more-distal of the two helices.

Some evidence has been provided that CbbX may "activate" Rubisco by a pore-threading mechanism (Mueller-Cajar et al. 2011). In this mechanism, Rubisco activase destabilizes Rubisco's closed, inactive conformation by moving the $\mathrm{C}$ terminus off of loop 6 , which then allows loop 6 to fold out of the active site (Duff et al. 2000). Although shorter in length than in the red-type Rubiscos, the $\mathrm{C}$ terminus of vascular-plant/green-algal Rubisco assumes a similar position in the closed conformation (Duff et al. 2000; Andersson 2008). In light of the significant structural differences between CbbX and Rubisco activase, including a major difference in the diameter of the central pore (Stotz et al. 2011), it remains unknown whether remodeling of the green-plant/algal Rubisco occurs by a similar mechanism.

It is currently unclear how a hexameric ring of Rubisco activase might interact with hexadecameric Rubisco to affect the $\mathrm{C}$ terminus, the active site, or the residues that determine species-specificity. All of these residues are located equatorially on the large subunit octamer (Fig. 1). In HslU/HslV (Zolkiewski 2006), another pore-threading AAA+ ATPase, the hexameric HslU ring caps the HslV proteolytic complex and unravels protein substrates. After unfolding, the polypeptide is then threaded through the central pore where it is hydrolyzed by the HslV protease. By analogy, Rubisco contains a central solvent channel that differs in diameter phylogenetically (Spreitzer 2003; Karkehabadi et al. 2005). The function of the solvent channel is unknown, but its presence might be necessary to accommodate the conformational changes induced by sugar-phosphate binding (Schreuder et al. 1993; Duff et al. 2000). These changes involve subtle domain reorientations coupled to active site transitions that are reversed by Rubisco activase.

A tetrad of small subunits caps the top and bottom of each Rubisco large-subunit octamer along the fourfold axis of symmetry (Fig. 1; Spreitzer 2003; Andersson and 

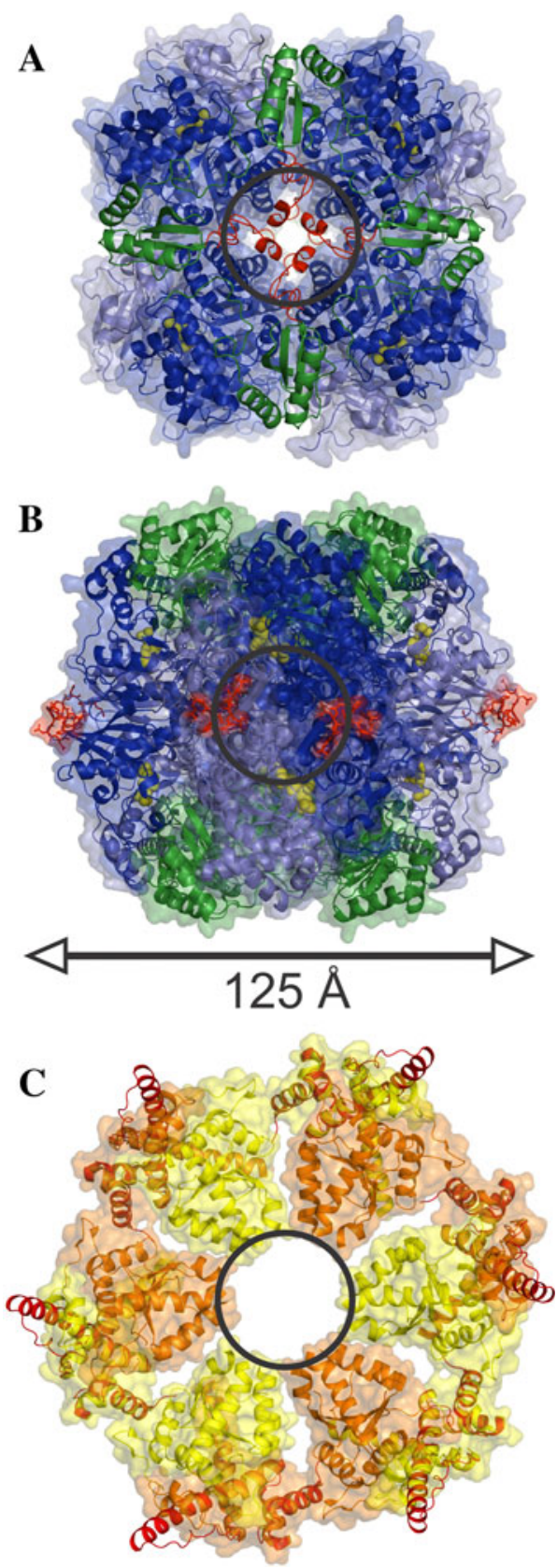

Fig. 1 Structural models of Rubisco and Rubisco activase. A Chlamydomonas Rubisco top view, and B side view (PDB 1GK8; Taylor et al. 2001). Shown are large subunits (blue), small subunits (green), pore-lining loops between $\beta$-strands $\mathbf{A}$ and $\mathbf{B}$ of the small subunit (red in $\mathbf{A}$ ), transition-state analogs (yellow), and recognition elements of the large subunit ( $r e d$ in B). C Closed-ring model of tobacco Rubisco activase, showing the hexameric AAA+ domain (PDB 3ZW6) in yellow and orange, with superimposed creosote activase recognition domains (PDB 3THG) in red. The circles shown are scaled to provide a diameter of $30 \AA$ in relation to the structures

Backlund 2008). The loop between small-subunit $\beta$-strands $\mathrm{A}$ and $\mathrm{B}$, which is variable in length, is oriented inward, and thus determines the diameter of the central solvent channel. If the initial interaction between Rubisco and
Rubisco activase involved a ring stacking mechanism that aligns the pore of the Rubisco activase hexamer with the Rubisco solvent channel, then the small subunits could comprise part of the contact interface between Rubisco and Rubisco activase.

Recently, Genkov et al. (2010) replaced the Chlamydomonas $r b c S$ genes that encode the small subunit of Rubisco with the $r b c S$ genes from spinach, Arabidopsis, or sunflower. All of the hybrid enzymes assembled and were functional in vivo, making it possible to examine the effects of foreign small subunits on the kinetic properties of the enzyme. However, the compatibility of these hybrid Rubiscos with plant Rubisco activases was not determined. Because all of the plant small subunits were from nonSolanaceae species, we constructed a hybrid Rubisco containing tobacco small subunits and Chlamydomonas large subunits to determine if the small subunits influence the species-specificity of the Rubisco-Rubisco activase interaction.

\section{Materials and methods ${ }^{1}$}

\section{Materials}

Biochemical reagents of the highest purity available were purchased from Sigma-Aldrich (St. Louis, MO). Radioactive $\mathrm{NaH}\left[{ }^{14} \mathrm{C}\right] \mathrm{CO}_{3}$ and $\left[2-{ }^{3} \mathrm{H}\right]$ glucose were purchased from PerkinElmer (Waltham, MA). Ribulose 1,5-bisphosphate was synthesized by isomerization and phosphorylation of ribose 5-phosphate (Jordan and Ogren 1984). [1- $\left.{ }^{3} \mathrm{H}\right]$ Ribulose 1,5-bisphosphate and phosphoglycolate phosphatase used in the assays were synthesized and purified as described previously (Jordan and Ogren 1981).

Algal strains and culture conditions

Cell-wall-less Chlamydomonas reinhardtii strain $r b c S \Delta$ T60-3 mt- was used as the host for $r b c S$ nuclear gene transformation as described previously (Khrebtukova and Spreitzer 1996; Genkov et al. 2010). The wild-type control strain WT-SS1, containing Chlamydomonas large and small subunits, as well as mutant strains SSAT, harboring a hybrid Rubisco containing the Chlamydomonas large and Arabidopsis small subunits, and SSSO, harboring a hybrid Rubisco containing the Chlamydomonas large and spinach small subunits, have been described previously (Genkov et al. 2010). For construction of mutant strain SSNT, a

\footnotetext{
${ }_{1}^{1}$ Mention of a trademark, proprietary product, or vendor does not constitute a guarantee or warranty of the product by the United States Department of Agriculture and does not imply its approval to the exclusion of other products or vendors that may also be suitable.
} 
codon-optimized sequence encoding the mature-protein coding region of Nicotiana tabacum rbcS (Fig. 2; Mazur and Chui 1985) was used to exactly replace the Chlamydomonas small-subunit coding region in plasmid pSS1ITP (Genkov et al. 2010). This new plasmid, named pSS1SSNT, was transformed into $r b c S \Delta-T 60-3$, and photosynthesis-competent colonies were recovered as described previously (Genkov et al. 2010). One of the transformant strains was used as the source of SSNT hybrid Rubisco. All strains were maintained at $25{ }^{\circ} \mathrm{C}$ in the dark with medium containing $10 \mathrm{mM}$ acetate and $1.5 \%$ Bacto-agar (Spreitzer and Mets 1981). For biochemical analysis, cells were grown in liquid acetate medium on a rotary shaker at $25^{\circ} \mathrm{C}$ in darkness. Cells were counted with a hemacytometer and harvested at the beginning of the stationary phase (about $10^{6}$ cells $\mathrm{mL}^{-1}$ ).

Isolation of Rubisco and Rubisco activase

Rubisco was isolated from tobacco leaves as described previously (Carmo-Silva et al. 2011). For isolation of Rubisco from Chlamydomonas, dark-grown cells were collected by centrifugation at $2,500 \times g$ for $5 \mathrm{~min}$. The cell pellets were suspended in an extraction buffer composed of $50 \mathrm{mM} N, N$-bis(2-hydroxyethyl) glycine (bicine), $\mathrm{pH} 8.0$, with $10 \mathrm{mM} \mathrm{NaHCO}, 10 \mathrm{mM} \mathrm{MgCl}$, and $1 \mathrm{mM}$ dithiothreitol, and were then sonicated at $0{ }^{\circ} \mathrm{C}$ for $3 \mathrm{~min}$. Cell debris were removed by centrifugation at $30,000 \times g$ for $15 \mathrm{~min}$, and Rubisco was isolated as described previously (Barta et al. 2010; Genkov et al. 2010). Recombinant tobacco and Arabidopsis Rubisco activase were isolated from Escherichia coli as described previously (Barta et al. 2011a).

Assay of Rubisco kinetics

The $V_{\mathrm{c}}$ and $K_{\mathrm{c}}$ kinetic constants of purified Rubisco were measured by the incorporation of ${ }^{14} \mathrm{CO}_{2}$ into acid-stable products in the absence of $\mathrm{O}_{2}$ (Chen et al. 1988). The ratio $(R)$ of Rubisco carboxylase activities measured in the absence and presence of $\mathrm{O}_{2}$ with varying concentrations of $\mathrm{CO}_{2}$ allows a direct determination of $K_{\mathrm{o}}$ according to the formula $R=1+K_{\mathrm{c}}\left[\mathrm{O}_{2}\right] / K_{\mathrm{o}}\left(K_{\mathrm{c}}+\left[\mathrm{CO}_{2}\right]\right.$ ) (Chen et al. $1988)$. The value of $\Omega\left(V_{\mathrm{c}} K_{\mathrm{o}} / V_{\mathrm{o}} K_{\mathrm{c}}\right)$ was determined from the ratio of the rates of carboxylase $\left(v_{\mathrm{c}}\right)$ and oxygenase $\left(v_{\mathrm{o}}\right)$ activities. These activities were measured simultaneously

\section{A}

ATGATGGTGTGGCCCCCCATCAACAAGAAGAAGTACGAGACCCTGTCCTACCTGCCCGACCTGTCCCAGGAGCAGCTGCT GTCCGAGGTCGAGTACCTGCTGAAGAACGGCTGGGTGCCCTGCCTGGAGTTCGAGACCGAGCACGGCTTCGTGTACCGCG AGAACAACAAGTCCCCCGGCTACTACGACGGCCGCTACTGGACCATGTGGAAGCTGCCCATGTTCGGCTGCACCGACGCC ACCCAGGTGCTGGCCGAGGTCGAGGAGGCCAAGAAGGCCTACCCCCAGGCCTGGATCCGCATCATCGGCTTCGACAACGT GCGCCAGGTGCAGTGCATCTCCTTCATCGCCTACAAGCCCGAGGGCTACTAA

B
10
20
30
40
50
60
70

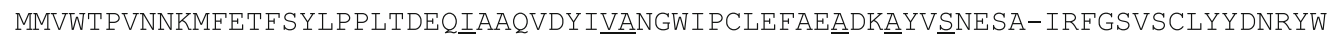

...PTQ.M.RY..L......TD.L.R....LN.K.V.....T-.HGF.YR.HHN------.PG...G..

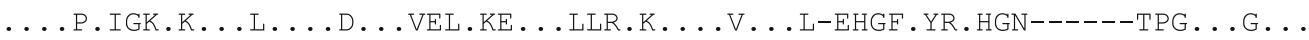

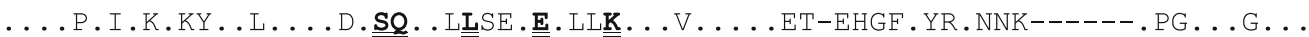

$\begin{array}{rrrrrr}80 & 90 & 100 & 110 & 120 & 130\end{array}$

TMWKLPMFGCRDPMQVLREIVACTKAFPDAYVRLVAFDNQKQVQIMGFLVQRPKTARDFQPANKRSV

$$
\begin{aligned}
& \text {.........A...N.LEE.K.EY.N.FI.IIG..SNR...CVS.IAYK.AGY----------- spinach } \\
& \text {........T.SA...K.VEE.K.EY.G.FI.IIG...TR...CIS.IAYK.PSET.A-------- Arabidopsis }
\end{aligned}
$$

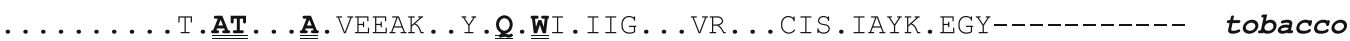

Fig. 2 Comparison of Rubisco small-subunit sequences. A The $r b c S$ coding sequence for the mature small subunit of tobacco was optimized for expression in Chlamydomonas, and engineered to replace Gln2 with Met2 to facilitate transit-peptide processing. B Comparison of the amino acid sequences of the Chlamydomonas, spinach, Arabidopsis, and tobacco Rubisco small subunits. Solvent- exposed residues of the Chlamydomonas small subunit that differ from those in the vascular-plant small subunits are underlined. Solvent-exposed residues of the tobacco small subunit that differ from those in Chlamydomonas, spinach, and Arabidopsis are in bold face and are underlined with two lines 
with $88 \mu \mathrm{M} \quad\left[1-{ }^{3} \mathrm{H}\right] \mathrm{RuBP} \quad\left(15.8 \mathrm{Ci} \mathrm{mol}^{-1}\right)$ and $2 \mathrm{mM}$ $\mathrm{NaH}^{14} \mathrm{CO}_{3}\left(0.5 \mathrm{Ci} \mathrm{mol}^{-1}\right)$ in 30 -min reactions at $25{ }^{\circ} \mathrm{C}$. The specificity factor, $\Omega$, was determined from the formula $\Omega=v_{\mathrm{c}} / v_{\mathrm{o}} \times\left[\mathrm{CO}_{2}\right] /\left[\mathrm{O}_{2}\right]$ (Jordan and Ogren 1981; Spreitzer et al. 1982).

\section{Assay of Rubisco activation}

Purified Rubisco, stored at $-80{ }^{\circ} \mathrm{C}$ as a frozen $65 \%$ ammonium sulfate suspension, was thawed, and the protein was collected by centrifugation at $10,000 \times g$ for $10 \mathrm{~min}$. The protein pellet was dissolved in $100 \mathrm{mM}$ Tricine$\mathrm{NaOH}, \mathrm{pH} 8.0,10 \mathrm{mM} \mathrm{MgCl} 2,10 \mathrm{mM} \mathrm{NaHCO}$, and $50 \mathrm{mM}$ dithiothreitol and incubated on ice for $60 \mathrm{~min}$. The solution was desalted by centrifugal gel-filtration in columns containing Sephadex-G50 that were equilibrated with $20 \mathrm{mM}$ Tricine- $\mathrm{NaOH}, \mathrm{pH} 8.0$, and $0.2 \mathrm{mM}$ EDTA to decarbamylate Rubisco (Carmo-Silva et al. 2011). The desalted protein was supplemented with $0.5 \mathrm{mM} \mathrm{RuBP}$ and incubated overnight at $4{ }^{\circ} \mathrm{C}$ to produce uncarbamylated enzyme with RuBP bound (i.e., ER form of the enzyme). Activation of this form of WT-SS1 and hybrid Chlamydomonas Rubisco with various amounts of purified recombinant Arabidopsis or tobacco Rubisco activase was determined in a time-course experiment as described previously (Barta et al. 2011b). The rate of Rubisco activation by Rubisco activase was determined during the linear phase of the time-course and is expressed as the fraction of Rubisco active sites that were restored to catalytic competence per min. This fraction was determined by comparison with the activity of the fully carbamylated enzyme (Carmo-Silva and Salvucci 2011).

\section{Results and discussion}

Sequence comparison of Chlamydomonas and plant Rubisco small subunits

Analysis of a set of sequences indicates that the Chlamydomonas small subunits exhibit about $45 \%$ sequence identity with plant small subunits whereas among plant small subunits sequence identity is about $70 \%$ (Spreitzer 2003). The amino acid sequence of the Chlamydomonas small subunit is 48,45 , and $44 \%$ identical with the small-subunit sequences of spinach, Arabidopsis, and tobacco, respectively (Fig. 2). Many of the residues that differ between Chlamydomonas and vascular-plant small subunits are solvent exposed (Genkov et al. 2010). However, when examining sequence alignments to identify solvent-exposed residues that are conserved between Chlamydomonas and non-Solanaceae plants but differ in the Solanaceae plant tobacco (Fig. 2), only conservative substitutions could be identified.
Replacements such as Thr to Ser or Ala to Leu are less likely to serve as recognition elements for species-specificity than more drastic substitutions. Therefore, it appears unlikely that solvent-exposed small-subunit residues dominate the molecular interactions between Rubisco activase subunits and their cognate Rubiscos.

Sequence comparisons alone cannot be used to exclude the possibility of molecular contact between the Rubisco small subunits and functional Rubisco activase assemblies during the Rubisco reactivation process. Therefore, surface calculations using the program AREAIMOL (CCP4 1994) were carried out on a coordinate file for the Chlamydomonas Rubisco holoenzyme (PDB 1G8K; Taylor et al. 2001) without solvent molecules. This analysis revealed that the small subunit comprises $32.2 \%$ of the solventaccessible surface area of the holoenzyme $\left(118,516 \AA^{2}\right)$. More than three quarters of the small-subunit surface $\left(76.8 \%, 4,773 \AA^{2}\right)$ is solvent-exposed whereas only $1,442 \AA^{2}$ are occluded by van der Waals contact with the large subunits. These values demonstrate that ample opportunity exists for direct interactions between supramolecular complexes of Rubisco activase and the Rubisco small subunits. Because the molecular dimensions of a Rubisco activase hexamer and Rubisco holoenzyme are comparable, it is difficult to imagine that binding involves no contact with the small subunits (Fig. 1).

Hybrid Rubiscos with Chlamydomonas large subunits and plant small subunits

In a previous study, hybrid Rubisco enzymes composed of Chlamydomonas large subunits and small subunits from spinach (SSSO), Arabidopsis (SSAT), or sunflower (SSHA) supported photoautotrophic growth of Chlamydomonas in air, albeit at a slower rate than the wild-type enzyme (Genkov et al. 2010). Compared with wild-type Chlamydomonas Rubisco, the hybrid enzymes had increased $\mathrm{CO}_{2} / \mathrm{O}_{2}$ specificity $(\Omega)$ and near-normal $V_{\mathrm{c}}$ values, but the cells expressing hybrid Rubisco lacked a pyrenoid. Because localization of Rubisco in the pyrenoid is a major requirement for the $\mathrm{CO}_{2}$ concentrating mechanism (Moroney and Ynalvez 2007; Spalding 2008), its absence from the hybrid-enzyme mutants explains their slower growth in air (Genkov et al. 2010).

Previous studies have shown that Rubisco activase from non-Solanaceae species, like spinach, readily activates Chlamydomonas Rubisco, but Rubisco activase from tobacco and other Solanaceae exhibits almost no ability to activate the algal Rubisco (Larson et al. 1997; Ott et al. 2000) or Rubisco from non-Solanaceae vascular plants (Wang et al. 1992). To determine if the small subunit of Rubisco affects the activation of Rubisco by Rubisco activase, a hybrid-mutant strain, SSNT, was constructed by 
replacing the Chlamydomonas small subunits with the small subunits from tobacco. As with the other Rubisco hybrid mutants (Genkov et al. 2010), SSNT grew slowly when cultured photoautotrophically in air, but growth was near wild-type levels when provided with $5 \% \mathrm{CO}_{2}$ in air (data not shown). Growth in $5 \% \mathrm{CO}_{2}$ would compensate for the lack of a pyrenoid (Genkov et al. 2010).

Kinetic properties of hybrid tobacco-Chlamydomonas Rubisco

The kinetic properties of the SSNT hybrid Rubisco were determined after purification of the enzyme from dark-grown cells. As with the other Rubisco hybrid enzymes previously analyzed (Genkov et al. 2010), the $\Omega$ value of SSNT Rubisco was greater than that of wild-type Chlamydomonas Rubisco (WT-SS1), but lower than tobacco Rubisco (Table 1). The $V_{\mathrm{c}}$ of the SSNT hybrid Rubisco was lower than that of wildtype Chlamydomonas Rubisco, but higher than tobacco Rubisco, whereas the affinity of the hybrid enzyme for $\mathrm{CO}_{2}$ $\left(K_{\mathrm{c}}\right)$ was more similar to Chlamydomonas Rubisco (i.e., lower than for the plant enzyme). In summary, the kinetic properties of the SSNT hybrid Rubisco were similar to the properties of the other hybrid Rubisco enzymes containing either spinach, Arabidopsis, or sunflower small subunits and Chlamydomonas large subunits (Genkov et al. 2010).

Activation of hybrid Rubisco by Rubisco activases from different species

Genkov et al. (2010) noted that, in addition to defective pyrenoid formation, the slower photoautotrophic growth of Chlamydomonas cells containing hybrid Rubiscos might be a consequence of impaired Rubisco activation by the native Rubisco activase. The hybrid Rubisco enzymes created in that study contained small subunits from non-Solanaceae species. Normally, Rubisco from these species is compatible with Chlamydomonas Rubisco activase (Wang et al. 1992), but a hybrid Rubisco composed of plant small subunits and Chlamydomonas large subunits might be impaired in its interaction with the endogenous Chlamydomonas Rubisco activase. A tobacco-Chlamydomonas hybrid is an even better enzyme for investigating the effect of small subunits on the interaction with Rubisco activase because tobacco Rubisco activase is incompatible with Chlamydomonas Rubisco (Wang et al. 1992; Ott et al. 2000; Li et al. 2005).

The rate of activation of the SSNT, SSSO, and SSAT hybrid Rubiscos, as well as Chlamydomonas wild-type (WTSS1) Rubisco, was determined in the presence and absence of recombinant Arabidopsis and tobacco Rubisco activase. Arabidopsis Rubisco activase, a good activator of Chlamydomonas Rubisco, was used because we were unable to express Chlamydomonas Rubisco activase in an active form. Without Rubisco activase, the rate of spontaneous activation of the inactive form of these enzymes, complexed with RuBP, was negligible (Fig. 3), indicating that all of the hybrid Rubisco enzymes were able to bind RuBP tightly when in the uncarbamylated form. In contrast, all three of the hybrid enzymes, as well as WT-SS1, activated when incubated with recombinant Arabidopsis Rubisco activase and ATP. The rate of activation of the ER form of these enzymes increased with increasing concentration of Arabidopsis Rubisco activase, approaching the rate determined in the absence of RuBP (i.e., $\mathrm{ECM})$ at the highest value tested, $5.8 \mu \mathrm{M}(0.25 \mathrm{mg} / \mathrm{ml}) \mathrm{Ru}-$ bisco activase subunit concentration. At a given concentration of Rubisco activase, the rate of activation of the hybrid enzymes was similar to the rate measured for WT-SS1 Rubisco. Also, the rate of spontaneous activation of the hybrid enzymes, measured in the absence of RuBP, i.e., ECM, was also similar to wild type (Fig. 3). When recombinant tobacco Rubisco activase was used, the rate of activation of wild-type and hybrid Rubisco was only slightly greater than the very slow spontaneous rate measured in the absence of Rubisco activase. At the concentrations used here, the tobacco Rubisco activase is able to activate the ER form of tobacco Rubisco at rates similar to those observed for the Arabidopsis enzyme (Salvucci 1992; Wang et al. 1992).

Implication of the results for the interaction of Rubisco activase with Rubisco

The hybrid Rubiscos composed of vascular-plant small subunits and Chlamydomonas large subunits were activated by Arabidopsis Rubisco activase at the same rate as the

Table 1 Kinetic properties of a hybrid Rubisco composed of tobacco (Nicotiana tabacum) small subunits and Chlamydomonas large subunits (SSNT), and the corresponding wild-type enzymes from Chlamydomonas (WT-SS1) and tobacco

\begin{tabular}{|c|c|c|c|c|c|c|c|}
\hline Rubisco & $\Omega^{\mathrm{a}}\left(V_{\mathrm{c}} K_{\mathrm{o}} / V_{\mathrm{o}} K_{\mathrm{c}}\right)$ & $V_{\mathrm{c}}^{\mathrm{a}}\left(\mu \mathrm{mol} \mathrm{h}{ }^{-1} \mathrm{mg}^{-1}\right)$ & $K_{\mathrm{c}}^{\mathrm{a}}\left(\mu \mathrm{M} \mathrm{CO} \mathrm{CO}_{2}\right)$ & $K_{\mathrm{o}}^{\mathrm{a}}\left(\mu \mathrm{M} \mathrm{O} \mathrm{O}_{2}\right)$ & $V_{\mathrm{c}} / K_{\mathrm{c}}^{\mathrm{b}}$ & $K_{\mathrm{o}} / K_{\mathrm{c}}^{\mathrm{b}}$ & $\overline{V_{\mathrm{c}} / V_{\mathrm{o}}^{\mathrm{b}}}$ \\
\hline Hybrid SSNT & $66 \pm 2$ & $86 \pm 9$ & $25 \pm 3$ & $439 \pm 44$ & 3.4 & 18 & 3.8 \\
\hline Chlamydomonas & $61 \pm 2$ & $111 \pm 6$ & $32 \pm 2$ & $510 \pm 8$ & 3.5 & 16 & 3.8 \\
\hline Tobacco & $76 \pm 1$ & $62 \pm 1$ & $21 \pm 2$ & $600 \pm 30$ & 3.0 & 29 & 2.7 \\
\hline
\end{tabular}

${ }^{a}$ Values are the mean $\pm \mathrm{SD}(n-1)$ of measurements with three separate enzyme preparations

${ }^{\mathrm{b}}$ Calculated values 
Chlamydomonas wild-type Rubisco. Similarly, replacing the Chlamydomonas small subunits with small subunits from tobacco did not improve the compatibility of Chlamydomonas Rubisco with tobacco Rubisco activase. Taken together, the most likely explanation for these results is that the interaction of Rubisco activase with Rubisco does not involve determinants on the Rubisco small subunit.

In a previous study, Kanevski et al. (1999) used plastome engineering in tobacco to produce a hybrid Rubisco enzyme composed of large subunits from sunflower, a nonSolanaceae species, and small subunits from tobacco. The engineered tobacco plants grew poorly in air because of the

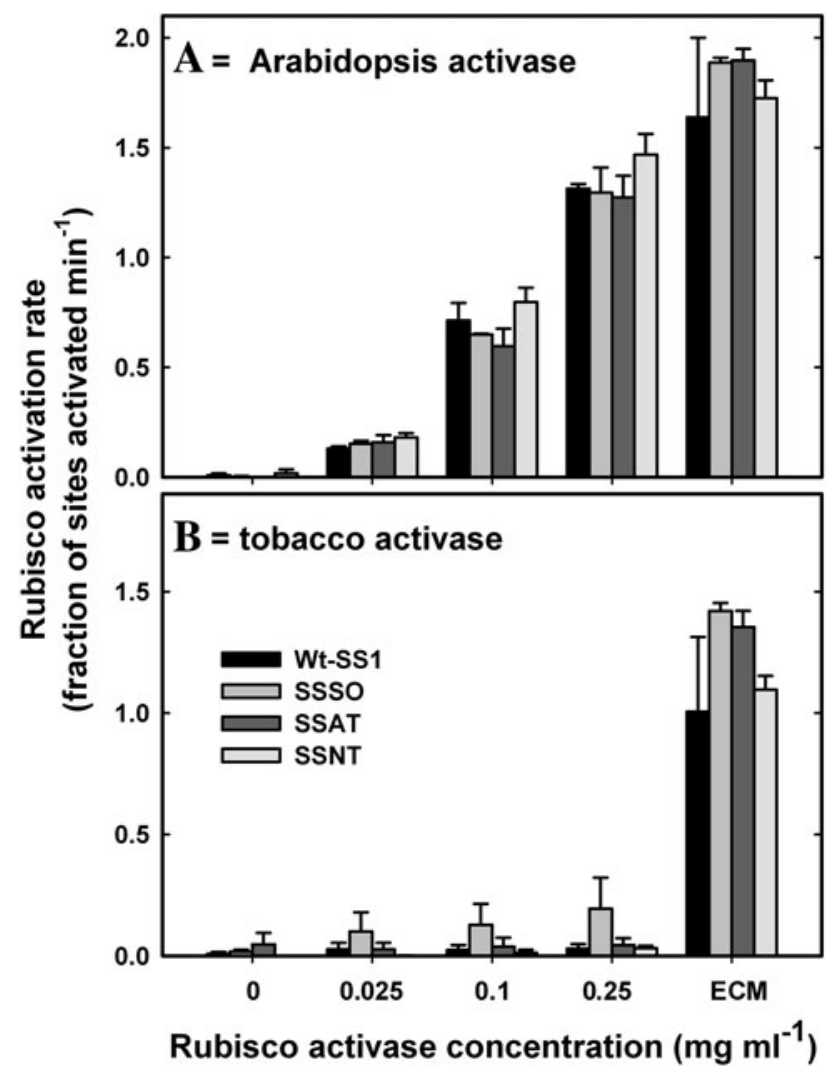

Fig. 3 Activation of purified Chlamydomonas wild type (WT-SS1) and hybrid Rubisco mutants containing the small subunits of Arabidopsis (SSAT), spinach (SSSO), and tobacco (SSNT) with Rubisco activase. Uncarbamylated Rubisco complexed with RuBP at $0.7 \mathrm{mg} \mathrm{ml}^{-1}$ was incubated with the indicated concentration of purified recombinant Arabidopsis (A) and tobacco (B) Rubisco activase and ATP. The rate of Rubisco activation was determined in time-course experiments, and was based on the activity of fully carbamylated enzyme (Carmo-Silva and Salvucci 2011). Rates for the fully activated enzyme (ECM) were determined in the absence of RuBP and with the highest concentration of Rubisco activase. The specific activity of the ECM Rubisco was $2.42 \pm 0.29,2.25 \pm 0.29$, $1.63 \pm 0.22$ and $1.56 \pm 0.22$ for the WT-SS1, SSAT, SSSO, and SSNT Rubiscos, respectively. The specific activity of the ER Rubisco (zero Rubisco activase) was $0.33 \pm 0.07,0.28 \pm 0.05,0.19 \pm 0.03$, and $0.25 \pm 0.07$ for the WT-SS1, SSAT, SSSO, and SSNT Rubiscos, respectively reduced level of expression of Rubisco (Sharwood et al. 2008). Although activation of the sunflower-tobacco hybrid Rubisco by tobacco Rubisco activase was not examined directly, Sharwood et al. (2008) concluded that the hybrid Rubisco was activated by the endogenous tobacco Rubisco activase based on measurements of Rubisco carbamylation and photosynthetic induction. The authors postulated that the four- to sevenfold greater stoichiometry of Rubisco activase to Rubisco in these plants was sufficient to overcome the incompatibility of tobacco Rubisco activase with non-Solanaceae Rubisco. While this explanation is certainly plausible, it is also possible that the looser binding of RuBP by the hybrid Rubisco, indicated by the fivefold increase in the $K_{\mathrm{m}}$ for RuBP (Kanevski et al. 1999), might have eliminated the requirements for Rubisco activase by allowing for spontaneous activation of Rubisco.

We recognize that our data with hybrid Rubisco do not preclude the possibility that residues of the small subunit participate in the interaction with Rubisco activase. Residues or structural regions involved in the interaction could be similar enough in vascular plants and Chlamydomonas to preserve Rubisco activation and species specificity. This possibility seems remote considering that, of the 41 solvent-exposed small-subunit residues in Chlamydomonas Rubisco (Genkov et al. 2010), 20 differ between Chlamydomonas and the three plant species used in this study. In addition, the tobacco small subunit has ten solventexposed residues that differ from the corresponding residues in Chlamydomonas, Arabidopsis, and spinach (Fig. 2).

The inability of the hybrid Rubiscos to form a pyrenoid in Chlamydomonas indicates that the small-subunit surface of Chlamydomonas Rubisco changes significantly when the endogenous small subunits are replaced with subunits from vascular plants (Genkov et al. 2010). This hypothesis was recently verified by the engineering of solvent-exposed small-subunit $\alpha$-helices (Meyer et al. 2012). If the small subunits were, in fact, involved in an interaction with Rubisco activase, then changes in the small-subunit structure significant enough to affect pyrenoid formation would also be expected to affect the interaction with Rubisco activase.

\section{Stoichiometry of the interaction}

Although the stoichiometry of productive interactions between Rubisco activase and Rubisco remain unknown, hexameric assemblies are thought to be critical for this process (Stotz et al. 2011). In the reactivation assays reported here, the molar ratio of Rubisco activase subunits to holo-Rubisco was 4.45 at the highest concentration of Rubisco activase tested $(5.8 \mu \mathrm{M})$, where high reactivation rates are observed (Fig. 3). At this concentration, Rubisco 
activase is thought to be composed of roughly equal concentrations of monomer, dimer, tetramer and hexamer, as demonstrated by use of fluorescence fluctuation methods (Chakraborty et al. 2012). However, at the lowest concentration examined $(0.58 \mu \mathrm{M})$, Rubisco activase is thought to consist of roughly $80 \%$ monomer and $20 \%$ dimer, with negligible concentrations of hexameric species. At this concentration of Rubisco activase, the rates of Rubisco reactivation were much slower than with higher concentrations (Fig. 3). Currently, quantitative assembly data are available only for ADP-bound Rubisco activase (Chakraborty et al. 2012), but other data indicate that the presence of ATP promotes Rubisco activase assembly to a more highly associated state at lower protein concentrations (Wang et al. 1993). Such a scenario would provide a rationale for the observed reactivation rates at $0.58 \mu \mathrm{M}$ Rubisco activase.

Hypothetical interaction models of Rubisco activase with Rubisco

For red-type Rubiscos, a model has been proposed involving the threading of the Rubisco large-subunit $\mathrm{C}$ terminus through the central pore of hexameric activase rings (Mueller-Cajar et al. 2011). Experimental evidence for a similar mechanism in green-type Rubiscos has not yet been provided. Thus, the precise mechanisms of recognition, interaction, and activation for Rubisco activase with Rubisco are unknown.

Without prior knowledge, two fundamental interaction geometries may be distinguished. One hypothetical binding mode involves the stacking of a Rubisco activase hexameric ring onto the fourfold ring of the Rubisco holoenzyme such that the sixfold and fourfold axes are aligned with each other (Fig. 4A). This "top-on" model based on a ring-ring stacking mechanism would invoke substantial molecular contacts with the Rubisco small subunits. Ample precedent exists for the stacking of closed AAA+ rings onto multi-subunit rings of partner proteins or partner domains with either matched or mismatched stoichiometry. Examples are HslU-HslV (Wang et al. 2001), FtsH (Bieniossek et al. 2006), ClpAP (Kress et al. 2007), and the proteasomal ATPase (Smith et al. 2007; Rabl et al. 2008). Peptide targets to be sequestered into the pore of a Rubisco activase ring may consist of loop regions surrounding the entrance of the Rubisco solvent channel, or segments of the Rubisco large subunits exposed between the small subunits (Fig. 1A). Along these lines, a hypothetical contact mode may involve a large-subunit helical hairpin comprising helices $\mathrm{G}$ and $\mathrm{H}$ immediately preceding the $\mathrm{C}$-terminal tail involved in active-site occlusion.

An alternate hypothetical binding mode involves a "side-on" model as shown in Fig. 4B. A Rubisco activase hexameric toroid may bind near the middle of Rubisco such that its central pore is centered over the twofold rotation axis of a functional large-subunit dimer (the center of the circle shown in Fig. 1B). In this orientation, the holoenzyme fourfold rotation axis would be positioned essentially perpendicular to the pseudo-sixfold axis of Rubisco activase assemblies. This binding mode would allow for the positioning of two Rubisco active sites and two Rubisco recognition elements near the central pore edge of the Rubisco activase hexamer, where mobile Rubisco activase loops essential for reactivation are located. This model involves substantial molecular contact with the Rubisco large subunits and minimal contact with the small subunits, which is supported by the data presented here.

With minor modifications, the side-on model could also accommodate the centering of Rubisco activase over one of
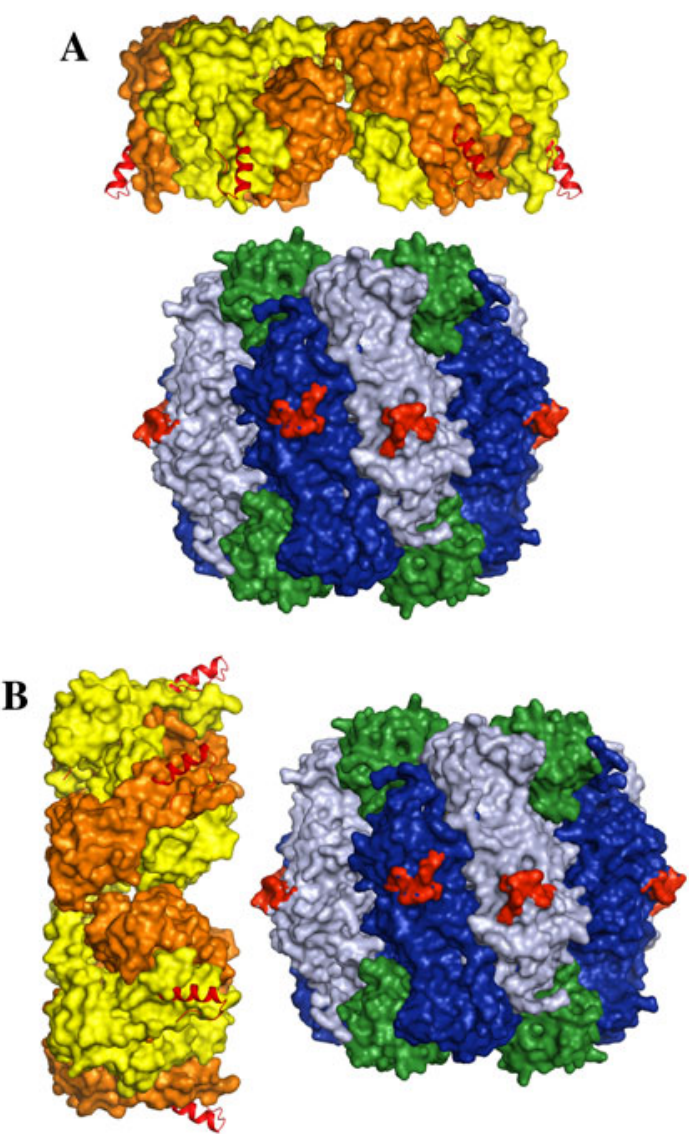

Fig. 4 Schematic of two Rubisco-Rubisco activase interaction models. A Top-on model based on ring-ring stacking, indicating substantial molecular contacts with the Rubisco small subunit, and B side-on model based on positioning two Rubisco active sites and two Rubisco recognition elements near the central pore edge of the toroidal activase hexamer as indicated in Fig. 1. The side-on model indicates substantial molecular contact of Rubisco activase with the Rubisco large subunit and minimal contact with the small subunit as supported by the data presented in this study 
the Rubisco active sites as was proposed for $\mathrm{CbbX}(\mathrm{Mu}-$ eller-Cajar et al. 2011). In this way, the $C$ terminus of the large subunit could be sequestered into the central pore of Rubisco activase. The sequestration of disordered protein segments has been demonstrated for a number of AAA+ assemblies such as FtsH (Bieniossek et al. 2006), the proteasomal ATPase (Smith et al. 2007), spastin, and ClpA/B (Kress et al. 2007).

Regardless of the specific binding interactions, neither of the two fundamental geometric arrangements appears to bring the Rubisco activase recognition motifs into proximity of their cognate sites on Rubisco (Fig. 4). However, the relevant arrangements of the $\mathrm{N}$ - and $\mathrm{C}$-terminal domains of the Rubisco activase AAA+ module remain unknown because hinge-bending motions near the bridge linking these domains are thought to be correlated with catalytic cycles. It should be noted that the closed-ring model shown in Figs. 1 and 4 was originally generated by fitting the X-ray structures of the two Rubisco activase domains into EM maps using FtsH as a template (Stotz et al. 2011). Hexameric toroidal assemblies may undergo significant shape modifications because the AAA+ C-domains may reorient as a function of nucleotide hydrolysis.

Currently available X-ray crystallographic data provide for a spiral subunit packing arrangement within the crystal with unknown physiological relevance (Stotz et al. 2011). The production of long fibrillar structures by CbbX, the red-type Rubisco activase, suggests that this spiral packing of subunits might be responsible for the very large molecular mass complexes often reported for Rubisco activase in solution (Barta et al. 2010; Chakraborty et al. 2012; Henderson et al. 2013). It is likely that the very high concentration of protein in the chloroplast stroma, including Rubisco at nearly $0.5 \mathrm{mM}$, would favor association with Rubisco over fibrillation.

Based on the data provided in this study, we favor the "side-on" over the "top-on" model (Fig. 4) because the species-specific interaction between Rubisco activase and Rubisco was not altered in the SSNT hybrid Rubisco enzyme. This observation makes it unlikely that the interaction between the two proteins involves a ring stacking mechanism that depends on the small subunit for docking and orientation via the central solvent channel.

Acknowledgments This study was funded by the Division of Chemical Sciences, Geosciences, and Biosciences, Office of Basic Energy Sciences, of the United States Department of Energy through Photosynthetic Systems Grants DE-FG02-00ER15044 to R.J.S., DEFG02-09ER16123 to R.M.W. and DE-AI02-97ER20268 to M.E.S. The authors would like to thank Professor Govindjee for his many contributions to photosynthesis including encouraging publication of the first report of Rubisco activase in Photosynthesis Research 7: 193-201 (1985).

\section{References}

Andersson I (2008) Catalysis and regulation in Rubisco. J Exp Bot 59:1555-1568

Andersson I, Backlund A (2008) Structure and function of Rubisco. Plant Physiol Biochem 46:275-291

Andrews TJ (1996) The bait in the Rubisco mousetrap. Nat Struct Biol 3:3-7

Barta C, Dunkle AM, Wachter RM, Salvucci ME (2010) Structural changes associated with the acute thermal instability of Rubisco activase. Arch Biochem Biophys 499:17-25

Barta C, Carmo-Silva AE, Salvucci ME (2011a) Purification of Rubisco activase from leaves or after expression in Escherichia coli. In: Carpentier R (ed) Photosynthesis Research Protocols Methods in Molecular Biology, vol 684. Human Press, New York, pp 363-374

Barta C, Carmo-Silva AE, Salvucci ME (2011b) Rubisco activase activity assays. In: Carpentier R (ed) Photosynthesis Research Protocols Methods in Molecular Biology, vol 684. Human Press, New York, pp 375-382

Bieniossek C, Schalch T, Bumann M, Meister M, Meier R, Baumann $U$ (2006) The molecular architecture of the metalloprotease FtsH. Proc Natl Acad Sci USA 103:3066-3071

Blankenship RE (2002) Molecular mechanisms of photosynthesis. Blackwell Science, London, p 321

Carmo-Silva AE, Salvucci ME (2011) The activity of Rubisco's molecular chaperone, Rubisco activase, in leaf extracts. Photosynth Res 108:143-155

Carmo-Silva AE, Barta C, Salvucci ME (2011) Isolation of ribulose1,5-bisphosphate carboxylase/oxygenase from leaves. In: Carpentier R (ed) Photosynthesis research protocols, methods in molecular biology, vol 684. Human Press, New York, pp 339-347

CCP4 (1994) The CCP4 suite: programs for protein crystallography. Acta Cryst D 50:760-763

Chakraborty M, Kuriata AM, Henderson JN, Salvucci ME, Wachter RM, Levitus M (2012) Protein oligomerization monitored by fluorescence fluctuation spectroscopy: self-assembly of Rubisco activase. Biophys J 103:949-958

Chen ZX, Chastain CJ, Al-Abed SR, Chollet R, Spreitzer RJ (1988) Reduced $\mathrm{CO}_{2} / \mathrm{O}_{2}$ specificity of ribulose-bisphosphate carboxylase/oxygenase in a temperature-sensitive chloroplast mutant of Chlamydomonas. Proc Natl Acad Sci USA 85:4696-4699

Duff AP, Andrews TJ, Curmi PMG (2000) The transition between the open and closed states of Rubisco is triggered by the interphosphate distance of the bound bisphosphate. J Mol Biol 298:903-916

Genkov T, Meyer M, Griffiths H, Spreitzer RJ (2010) Functional hybrid Rubisco enzymes with plant small subunits and algal large subunits: engineered $r b c S$ cDNA for expression in Chlamydomonas. J Biol Chem 285:19833-19841

Henderson JN, Kuriata AM, Fromme R, Salvucci ME, Wachter RM (2011) Atomic resolution X-ray structure of the substrate recognition domain of higher plant ribulose-bisphosphate carboxylase/oxygenase (Rubisco) activase. J Biol Chem 286: 35683-35688

Henderson JN, Hazra S, Dunkle AM, Salvucci ME, Wachter RM (2013) Biophysical characterization of higher plant Rubisco activase. Biochim Biophys Acta 1834:87-97

Jordan DB, Ogren WL (1981) A sensitive assay procedure for simultaneous determination of ribulose-1,5-bisphosphate carboxylase and oxygenase activities. Plant Physiol 67:237-245

Jordan DB, Ogren WL (1984) The $\mathrm{CO}_{2} / \mathrm{O}_{2}$ specificity of ribulose $1,5-$ bisphosphate carboxylase/oxygenase-dependence on ribulosebisphosphate concentration, $\mathrm{pH}$ and temperature. Planta 161:308-313 
Jordan DB, Chollet R, Ogren WL (1983) Binding of phosphorylated effectors by active and inactive forms of ribulose-1,5-bisphosphate carboxylase. Biochemistry 22:3410-3418

Kanevski I, Maliga P, Rhoades DF, Gutteridge S (1999) Plastome engineering of ribulose-1,5-bisphosphate carboxylase/oxygenase in tobacco to form a sunflower large subunit and tobacco small subunit hybrid. Plant Physiol 119:133-141

Karkehabadi S, Peddi SR, Anwaruzzaman M, Taylor TC, Cederlund A, Genkov T, Andersson I, Spreitzer RJ (2005) Chimeric small subunits influence catalysis without causing global conformational changes in the crystal structure of ribulose-1,5-bisphosphate carboxylase/oxygenase. Biochemistry 44:9851-9861

Khrebtukova I, Spreitzer RJ (1996) Elimination of the Chlamydomonas gene family that encodes the small subunit of ribulose1,5-bisphosphate carboxylase/oxygenase. Proc Natl Acad Sci USA 93:13689-13693

Kress W, Mutschler H, Weber-Ban E (2007) Assembly pathway of an $\mathrm{AAA}+$ protein: tracking ClpA and ClpAP complex formation in real time. Biochemistry 46:6183-6193

Larson EM, O’Brien CM, Zhu G, Spreitzer RJ, Portis AR Jr (1997) Specificity for activase is changed by a Pro- 89 to Arg substitution in the large subunit of ribulose-1,5-bisphosphate carboxylase/oxygenase. J Biol Chem 272:17033-17037

Li C, Salvucci ME, Portis AR Jr (2005) Two residues of Rubisco activase involved in recognition of the Rubisco substrate. J Biol Chem 280:24864-24869

Mazur BJ, Chui CF (1985) Sequence of a genomic DNA clone for the small subunit of ribulose bisphosphate carboxylase-oxygenase from tobacco. Nucl Acids Res 13:2373-2386

Meyer MT, Genkov T, Skepper JN, Jouhet U, Mitchell MC, Spreitzer RJ, Griffiths H (2012) Rubisco small-subunit $\alpha$-helices control pyrenoid formation in Chlamydomonas. Proc Natl Acad Sci USA 109:19474-19479

Moroney JV, Ynalvez RA (2007) Proposed carbon dioxide concentrating mechanism in Chlamydomonas reinhardtii. Eukaryot Cell 6:1251-1259

Mueller-Cajar O, Stotz M, Wendler P, Hartl FU, Bracher A, HayerHartl M (2011) Structure and function of the AAA+ protein CbbX, a red-type Rubisco activase. Nature 479:194-199

Ott CM, Smith BD, Portis AR Jr, Spreitzer RJ (2000) Activase region on chloroplast ribulose-1,5-bisphosphate carboxylase/oxygenase: nonconservative substitution in the large subunit alters species specificity of protein interaction. J Biol Chem 275:26241-26244

Pearce FG (2006) Catalytic by-product formation and ligand binding by ribulose bisphosphate carboxylases from different phylogenies. Biochem J 399:525-534

Pearce FG, Andrews TJ (2003) The relationship between side reactions and the slow inhibition of ribulose-bisphosphate carboxylase revealed by a loop 6 mutant of the tobacco enzyme. J Biol Chem 278:32526-32536

Portis AR Jr (2003) Rubisco activase-Rubisco's catalytic chaperone. Photosynth Res 75:11-27

Portis AR Jr, Li C, Wang D, Salvucci ME (2008) Regulation of Rubisco activase and its interaction with Rubisco. J Exp Bot 59:1597-1604

Rabl J, Smith DM, Yu Y, Chang SC, Goldberg AL, Cheng Y (2008) Mechanism of gate opening in the $20 \mathrm{~S}$ proteasome by the proteasomal ATPases. Mol Cell 30:360-368

Salvucci ME (1992) Subunit interactions of Rubisco activase: polyethylene glycol promotes self-association, stimulates
ATPase and activation activities, and enhances interactions with Rubisco. Arch Biochem Biophys 298:688-696

Schreuder HA, Knight S, Curmi PM, Andersson I, Cascio D, Branden CI, Eisenberg D (1993) Formation of the active site of ribulose1,5-bisphosphate carboxylase/oxygenase by a disorder-order transition from the unactivated to the activated form. Proc Natl Acad Sci USA 90:9968-9972

Sharwood RE, von Caemmerer S, Maliga P, Whitney SM (2008) The catalytic properties of hybrid Rubisco comprising tobacco small and sunflower large subunits mirror the kinetically equivalent source Rubiscos and can support tobacco growth. Plant Physiol 146:83-96

Smith DM, Chang SC, Park S, Finley D, Cheng Y, Goldberg AL (2007) Docking of the proteasomal ATPase's carboxyl termini in the $20 \mathrm{~S}$ proteasome's alpha ring opens the gate for substrate entry. Mol Cell 27:731-744

Spalding MH (2008) Microalgal carbon-dioxide-concentrating mechanisms: Chlamydomonas inorganic carbon transporters. J Exp Bot 59:1463-1473

Spreitzer RJ (2003) Role of the Rubisco small subunit. Arch Biochem Biophys 414:141-149

Spreitzer RJ, Mets L (1981) Photosynthesis-deficient mutants of Chlamydomonas reinhardtii with associated light-sensitive phenotypes. Plant Physiol 67:565-569

Spreitzer RJ, Salvucci ME (2002) Rubisco: structure, regulatory interactions, and possibilities for a better enzyme. Annu Rev Plant Biol 53:449-475

Spreitzer RJ, Jordan DB, Ogren WL (1982) Biochemical and genetic analysis of an RuBP carboxylase/oxygenase-deficient mutant and revertants of Chlamydomonas reinhardtii. FEBS Lett 148:117-121

Stotz M, Mueller-Cajar O, Ciniawsky S, Wendler P, Hartl FU, Bracher A, Hayer-Hartl M (2011) Structure of green-type Rubisco activase from tobacco. Nature Struct Mol Biol 18: $1366-1370$

Taylor TC, Backlund A, Bjorhall K, Spreitzer RJ, Andersson I (2001) First crystal structure of Rubisco from a green alga, Chlamydomonas reinhardtii. J Biol Chem 276:48159-48164

Tcherkez GGB, Farquhar GD, Andrews TJ (2006) Despite slow catalysis and confused substrate specificity, all ribulose bisphosphate carboxylases may be nearly perfectly optimized. Proc Natl Acad Sci USA 103:7246-7251

Wang Z, Snyder GW, Esau BD, Portis AR Jr, Ogren WL (1992) Species-dependent variation in the interaction of substrate-bound ribulose-1,5-bisphosphate carboxylase/oxygenase (Rubisco) and Rubisco activase. Plant Physiol 100:1858-1862

Wang ZY, Ramage RT, Portis AR Jr (1993) $\mathrm{Mg}^{2+}$ and ATP or adenosine $5^{\prime}$-[ $\gamma$-thio]-triphosphate $(\mathrm{ATP} \gamma \mathrm{S})$ enhances intrinsic fluorescence and induces aggregation which increases the activity of spinach Rubisco activase. Biochim Biophys Acta 1202:47-55

Wang J, Song JJ, Franklin MC, Kamtekar S, Im YJ, Rho SH, Seong IS, Lee CS, Chung CH, Eom SH (2001) Crystal structures of the HslVU peptidase-ATPase complex reveal an ATP-dependent proteolysis mechanism. Structure 9:177-184

Whitney SM, Houtz RL, Alonso H (2011) Advancing our understanding and capacity to engineer nature's $\mathrm{CO}_{2}$-sequestering enzyme, Rubisco. Plant Physiol 155:27-35

Zolkiewski M (2006) A camel passes through the eye of a needle: protein unfolding activity of Clp ATPases. Mol Microbiol 61:1094-1100 